\title{
Oligosaccharides in infant formula
}

\author{
Y. Vandenplas* \\ Academic Children's Hospital, Free University of Brussels, Laarbeeklaan 101, 1090 Brussels, Belgium
}

\begin{abstract}
Breast-feeding is the golden standard for infant feeding. However, the majority of a few week old infants are fed with a second choice infant feeding, cow's milk based formula. Amongst the multiple differences between human and cow's milk regards the development of the gastrointestinal flora: the flora of the breast-fed infant being richer in bifidobacteria and lactobacilli. Both species are known to be potentially beneficial for the health of the host. The absence of oligosaccharides, the third largest component in human milk, in cow's milk is likely to account for the differences in colonic flora. The oligosaccharide content and concentration in breast milk is — just as for the other macronutrients — a dynamic process, making it impossible for industry to mimic nature. However, if the composition cannot be mimicked, the effect and function can be imitated. The addition of two oligosaccharides, galacto-oligosaccharides and inulin, to cow's milk based infant formula has been shown to have a bifidogenic effect, and to stimulate the growth of bifidi and lactobacilli. In conclusion, the addition of oligosaccharides to cow's milk based infant formula brings this alternative, second choice infant feeding one step closer to the golden standard of human milk. But, prolonged breast-feeding should still be promoted with maximum effort.
\end{abstract}

Bifidus bacteria: Breast-feeding: Inulin: Galacto-oligosaccharide: Human milk: Infant formula: Lactobacillus: Oligosaccharide

\section{Introduction}

Throughout European history, fermented milk products have been considered beneficial to health, but it is only in the recent years that sound scientific evidence has accumulated to support such a statement. Functional foods have resulted from the gradual recognition that healthy diets result from eating nutritious foods and from the identification of the mechanisms by which foods modulate metabolism and health (German et al. 1999). A growing number of food manufacturers in western Europe are exploring the commercial opportunities for foodstuffs containing healthpromoting microbial food supplements, probiotics, and health-promoting non-digestible food ingredients, prebiotics (Young, 1998). Probiotic bacteria and prebiotic carbohydrates or oligosaccharides have been demonstrated to have health benefits. The oligosaccharides inulin and oligofructose have recently attracted much attention as nondigestible carbohydrates with prebiotic properties (Jenkins et al. 1999). The molecular weight of these prebiotic oligosaccharides is of importance, since the longer the chain length the more sustained the fermentation pattern becomes (Jenkins et al. 1999).

\section{Oligosaccharides in human milk}

Human milk contains a high concentration of carbohydrates, especially oligosaccharides which are the third most abundant solid constituent of human milk (Newburg, 1996; Picciano, 2001). A function for non-immunologlobulin milk protective factors, many of them non-nutrients, in providing defence mechanisms to the baby is only now beginning to be detected. Prominent among postulated defence agents are the milk oligosaccharides and glycoconjugates. Their carbohydrate structures are thought to be assembled by the same enzymes, the glycosyltransferases that synthesise the cell surface glycoconjugates often used as receptors by pathogens. Human milk contains at least twenty-one different kinds of oligosaccharides composed of many different molecules some being linear, others being branched, some being composed of simple sugars like galactose others containing sugar derivatives like uronic acids or uronic este, some being acidic others being neutral.... (Coppa et al. 1999; Hamosh, 2001). It has been hypothesised that the variation in content in neutral oligosaccharides is independent of gestational age, duration of lactation and postconceptional age (Nakhla et al.

Note: For the definition of the terms inulin and oligofructose please refer to the introductory paper (p. S139) and its footnote.

* Corresponding author: Dr Y. Vandenplas, tel +32 247757 80, fax +32 247757 83, email yvan.vandenplas@az.vub.ac.be 
1999). However, many data contradict this observation. The highest amount of oligosaccharides, $20 \mathrm{~g} / \mathrm{l}$ milk, is reached on the fourth day of life (Coppa et al. 1999; Hamosh, 2001). On days 30 and 120 of lactation, there is decrease of $20 \%$ and $40 \%$, respectively, in comparison to day 4 (Coppa et al. 1993; 1999). The decrease in oligosaccharides is compensated by an increase in lactose content (Coppa et al. 1999). Not only does the amount of oligosaccharides in human milk differ in relation to the duration of lactation, but also the composition of oligosaccharides in human milk varies among different samples, and many factors combine for the final composition. The geographical origin of the mother is one of these factors (Erney et al. 2000). Whether the content and composition of oligosaccharides differ in the milk of a mother who delivered at term or preterm is a matter of debate. The quantitative individual variation in content of ten neutral oligosaccharides in preterm human milk is said to be comparable to the content in milk of mothers who delivered at term (Nakhla et al. 1999). However, these findings are contradicted by Coppa et al. (1997). These authors conclude that compared to term milk, preterm milk contains in the colostrum phase a significantly lower lactose concentration and a significantly higher oligosaccharide content. The faecal excretion of oligosaccharides by the baby decreases in relation to the decrease in breast-milk that is replaced by a formula not containing oligosaccharides (Sabharval et al. 1991). The pattern of oligosaccharides excreted in the urine by a breast-fed infant is related to the oligosaccharide profile in the mother's milk (Coppa et al. 1990).

There is substantial evidence that the oligosaccharide secretion in mother's milk is a complex, variable and dynamic process (Coppa et al. 1993). Therefore, as is the case for other macronutrients such as lipids, it should be the goal of an infant formula containing oligosaccharides to mimic the effect, but not the dynamic aspect of, the oligosaccharide content of mother's milk. Cow's milk, and thus infant formula, is very poor in oligosaccharides.

\section{The function of oligosaccharides in human milk}

Some milk oligosaccharides and glycoconjugates may protect the infant by acting as receptor homologues, inhibiting the binding of entero-pathogens to their host receptors. Neutral oligosaccharides cause inhibition of bacterial adhesion (Coppa et al. 1990). Breast-feeding may have a preventive effect on urinary tract infection in both the mother and the infant (Coppa et al. 1990). Ongoing research is linking specific carbohydrate structures with protection against specific pathogens.

Gluco-oligosaccharides ( $\alpha 1-2 ; \alpha 1-4 ; \alpha 1-6$ linkages) and maltodextrin-like oligosaccharides are nondigestible, yet fermentable oligosaccharides, and may act as 'dietary fibre like' ingredients (Flickinger et al. 2000). In order to avoid misunderstanding because of the terminology used, 'maltodextrin-like oligosaccharides' are different from 'maltodextrin', which is well digested via amylase. Gluco-oligosaccharides and maltodextrin-like oligosaccharides increase the numeric number and concentration of bifidobacteria in the faeces (Flickinger et al. 2000). However, supplementation of food with oligofructose (from sucrose) does not alter the cell counts of viable bifidobacterial organisms or total anaerobic microbiota, faecal $\mathrm{pH}$, or concentration of short-chain fatty acids (Howard et al. 1995). But, the number of bifidobacteria in the faeces is increased after 6 days (Howard et al. 1995). Data suggest that dietary consumption of oligofructose (from sucrose) will enhance population of bifidobacteria and prevent colonic epithelial mucosa atrophy in neonates fed an elemental diet (Howard et al. 1995). Total production of short-chain fatty acids is similar for oligofructose, gluco-oligosaccharides, guar gum, guar hydrolysate and gum arabic (Flickinger et al. 2000). These 'gums' are comparable to locust bean gum, used in some thickened anti-regurgitation formulas. (The prebiotic effect of some anti-regurgitation formulas has not been validated up to now.)

\section{Addition of pro- and/or prebiotics to infant formula}

Bifidobacteria, and to a lesser extent lactobacilli, are known to be potentially beneficial to the health of the host. Breast-fed infants are known to have a gastro-intestinal flora that is dominated by bifidobacteria and lactobacilli. And, although breast-fed and formula-fed infants have a similar gastro-intestinal flora on day 3 or 4 of life, there is a substantial difference in colonic flora after several weeks of life in function of feeding (Harmsen et al. 2000). Indeed, while bifidobacteria become predominant in breast-fed infants, the gastro-intestinal flora of formula-fed infants is dominated by anaerobic bacteria such as bacteroides and bifidobacteria (Harmsen et al. 2000). As a consequence, industry is evaluating methods to make the gastro-intestinal flora of formula-fed infants comparable to the flora of breast-fed infants. However, whether this different gastro-intestinal colonisation in young infants has clinically significant long-term consequences remains unclear. Anyway, it is unclear exactly how long these differences in gastro-intestinal colonisation persist, but after a few years, these differences have disappeared.

Some companies try to mimic gastro-intestinal colonisation of breast-fed infants by adding lactobacilli to the formula, while others try to mimic mother's milk and add oligosaccharides to the formula to obtain the same effect on gastro-intestinal colonisation as mother's milk.

The addition of lactobacilli to infant formula results in a gastro-intestinal flora that is dominated by lactobacilli, comparable to breast-fed infants (Langhendries et al. 1995). Not only does the flora becomes 'breast-fed like', but some typical aspects of breast-fed infants such as the aspect of stools change accordingly. These observations lead to the conclusion that the 'probiotic theory' is valid and that the development of the gastro-intestinal flora can be manipulated by adding lactobacilli to the infant formula. However, this concept may be regarded as 'unphysiological' since lactobacilli are - of course - not present in human milk. Lactobacilli may, however, be present on the skin of the breast of the lactating mother.... Another weakness in the probiotic concept is that the bacteria need to be administered in a viable way, necessitating specific guidelines of formula preparation. The addition of probiotics to infant formula has been shown to decrease 
the incidence and severity of episodes of infectious diarrhoea, such as rota-virus in hospitalised children (Saavedra, 2000).

In the prebiotic concept, oligosaccharides (inulin and/or oligofructose and galacto-oligosaccharides) are added to the infant formula. At first, this seems a more physiological approach. But, of course, the dynamic aspects of oligosaccharide content in mother's milk cannot be mimicked in artificial feeding. In the prebiotic concept, there are no special precautions or guidelines needed in the preparation of the formula. It has been shown that the addition of oligosaccharides to infant formula results in a similar effect on gastro-intestinal colonisation: the flora becomes predominant in bifidobacteria (Boehm et al. 2001). The absolute number of bifidobacteria and the proportion of bifidobacteria as a percentage of the total micro-organisms increase with a formula supplemented with prebiotics (Boehm et al. 2001; Knol et al. 2001). Consequently, the number of lactobacilli also increases significantly (Moro et al. 2001). Supplementation with bifidobacteria protects rats from developing necrotising enterocolitis. Equally, if the gastro-intestinal flora of a premature with necrotising enterocolitis is inoculated in gnotobiotic quails, the latter also develop necrotising enterocolitis. But, the necrotising enterocolitis does not develop if bifidobacteria are introduced simultaneously (Caplan \& Jilling, 2000). The growth and quality of growth of infants fed with a formula with prebiotics, $\beta$-palmitate and hydrolysed proteins were similar to those seen in breast-fed infants and current infant formulas (Boehm et al. 2001; Rigo et al. 2001).

\section{Theoretical health promoting effects of pro- and/or prebiotics}

A well-balanced, normal intestinal flora may have many health promoting effects. However, the scientific evidence for these effects is not equally solid for all. The intestinal wall of animals with a sterile gastro-intestinal tract is thinner than the wall of a gastro-intestinal tract with flora. This observation suggests that the flora has a role in offering protection against translocation and decreases gastro-intestinal permeability. The gastro-intestinal flora protects the host for colonisation with invasive micro-organisms. The flora regulates the gastro-intestinal transit and stimulates migrating motor complexes. The flora also has metabolic functions, since it intervenes in the deconjugation of bile salts and stimulates entero-hepatic circulation. Lactobacilli are capable of hydrolysing lactose that reaches the colon and thus has not been absorbed in the small bowel. The flora is capable of hydrolysing (or 'fermenting') complex carbohydrates that reach the colon. Short-chain fatty acids are produced as an end-product of this hydrolysis (Midtvedt, 1999). The flora also hydrolyses proteins and therefore may have a role in decreasing the allergenicity of non-digestible proteins.

Differences in the neonatal gut microflora precede the development of atopy, suggesting a crucial role of the balance of indigenous intestinal bacteria for the maturation of human immunity to a nonatopic mode (Kalliomaki et al. 2001). Allergic infants have an adult type Bifidobacterium flora with high levels of Bifidobacterium. Healthy infants have a typical infant Bifidobacterium flora with high levels of Bifidobacterium bifidum. The adhesion of the faecal bifidobacteria from healthy infants is significantly higher than those of allergic infants adolescentis (He et al. 2001). This suggests a correlation between allergic disease and the composition of the intestinal bifidobacteria flora which has reduced adhesive abilities to the intestinal mucus adolescentis (He et al. 2001). Oral administration of insoluble non-digestible polysaccharides induces a proliferation of IgA-producing B-lymphocytes in small intestinal and caecal mucosa in rats (Kudoh et al. 1998). The latter finding suggests that prebiotics may have a similar effect as probiotics on the decrease of allergenicity of dietary proteins. The long-term addition of a prebiotic mixture to cereals has been shown to be effective and safe (Saavedra et al. 1999; Tschernia et al. 1999).

\section{Side effects?}

Issues considered important to the continuing development of this growing market are proof of safety, proof of efficacy, consumer education, market positioning, price and appropriate health claim strategies. Oligosaccharides are in general considered as very safe. A recent meta-analysis of controlled short-term intervention studies shows that fermented yoghurt decreases total cholesterol level by $4 \%$ (Agerholm-Larsen et al. 2000). Lactobacillus reuteri given to mice even caused a decrease of cholesterol by $17 \%$ (Taranto et al. 2000). Also oligosaccharides are known to decrease cholesterol levels and to decrease weight. The effect of oligosaccharides in infant formula on plasma cholesterol has not been validated up to now. Although the data on weight gain are limited, weight gain in formula-fed infants with and without oligosaccharides seems comparable. In conclusion: although the safety aspects of oligosaccharides in infant formulas have not been sufficiently validated, it is highly probable that they can be considered as safe.

\section{Conclusion}

The addition of certain mixtures of oligosaccharides in infant formula bring infant formula, the second choice infant feeding, one step closer to the gold standard, breast- feeding. Although it seems impossible to mimic the complexity of human physiology, it seems that the beneficial health effects of oligosaccharides in human milk can be mimicked. However, more data are needed on the effect and safety on the addition of prebiotics (and probiotics) in infant formula before this can become generally applied.

\section{References}

Agerholm-Larsen L, Bell ML, Grunwald GK \& Astrup A (2000) The effect of a probiotic milk product on plasma cholesterol: a meta-analysis of short-term intervention studies. European Journal of Clinical Nutrition 54, 856-860.

Boehm G, Casetta P, Lidestri M, Negretti F, Jelinek J, Stahl J \& Marini A (2001) Effect of dietary oligosaccharides on faecal 
bifidobacteria in formula fed preterm infants. Journal of Pediatric Gastroenterology and Nutrition 32, 393.

Caplan MS \& Jilling T (2000) Neonatal necrotizing enterocolitis: possible role of probiotic supplementation. Journal of Pediatric Gastroenterology and Nutrition 30, suppl 2, S18-S22.

Coppa GC, Pierani P, Zampini L, Carloni I, Carlucci A \& Gabrielli O (1999) Oligosaccharides in human milk during different phases of lactation. Acta Paediatrica 88, suppl 430, 89-94.

Coppa GV, Gabrielli O, Girogi P, Catassi C, Montanari MP, Varaldo PE \& Nichols BL (1990) Preliminary study of breastfeeding and bacterial adhesion to uroepithelial cells. Lancet $\mathbf{3 3 5}$, $569-571$.

Coppa GV, Gabrielli O, Pierani P, Catasi C, Carlucci A \& Giorgi PL (1993) Changes in carbohydrate composition in human milk over 4 months of lactation. Pediatrics 91, 737-741.

Coppa GV, Pierani P, Zampini L, Gabrielli O, Carlucci A, Catassi C \& Giorgi P (1997) Lactose, oligosaccharide and monosacharide content of milk from mothers delivering preterm newborns over the first month of life. Minerva Pediatrica 49, 471-475.

Erney RM, Malone WT, Skelding MB, Marcon AA, KlemanLeyer KM, O'Ryan ML, Ruiz-palacios G, Hilty MD, Pickering LK \& Prieto PA (2000) Variability of human milk oligosaccharides in a diverse population. Journal of Paediatric Gastroenterology and Nutrition 30, 131-133.

Flickinger EA, Wolf BW, Galeb KA, Chow J, Leyer GJ, Johns PW \& Fahey GC (2000) Glucose-based oligosaccharides exhibit different in vitro fermentation patterns and affect in vivo apparent nutrient digestibility and microbial population in dogs. Journal of Nutrition 130, 1267-1273.

German B, Schiffrin EJ, Reniero R, Mollet B, Pfeifer A \& Neeser JR (1999) The development of functional foods: lessons from the gut. Trends in Biotechnology 17, 492-499.

Harmsen HJ, Wildeboer-Veloo AC, Raangs GC, Wagendorp AA, Klijn N, Bindels JG \& Welling GW (2000) Analysis of intestinal flora development in breast-fed and formula-fed infants by using molecular identification and detection methods. Journal of Pediatric Gastroenterology and Nutrition 30, 61-67.

He F, Ouwehand AC, Isolauri E, Hashimoto H, Benno Y \& Salminen S (2001) Comparison of mucosal adhesion and species identification of bifidobacteria isolated from healthy and allergic infants. FEMS Immunology Medicine and Microbiology 30, 43-47.

Howard MD, Gordon DT, Pace LW, garleb KA \& Kerley MS (1995) Effects of dietary supplementation with fructo-oligosaccharides on colonic microbiota populations and epithelial cell proliferation in neonatal pigs. Journal of Pediatric Gastroenterology and Nutrition 21, 297-303.

Jenkins DJ, Kendall CW \& Vuksan V (1999) Inulin, oligofructose and intestinal function. Journal of Nutrition 129, suppl 7, $1431-1433$.

Kalliomaki M, Kirjavainen P, Eerola E, Kero P, Salminen S \& Isolauri E (2001) Distinct patterns of neonatal gut microflora in infants in whom atopy was and was not developing. Journal of Allergy and Clinical Immunology 107, 129-134.

Knol J, Poellwijk ES, van der Linde EGM, Wells JCK, Brönstrup A, Kohlschmid N, Wirth S, Schmitz B, Skopnik H, Schmelzle H \& Fusch C (2001) Stimulation of endogenous bifidobacteria in term infants by an infant formula containing prebiotics. Journal of Pediatric Gastroenterology and Nutrition 32, 399.

Kudoh K, Shimizu J, Wada M, Takita T, Kanke Y \& Innami S (1998) Effect of indigestible saccharides on B lymphocyte response of intestinal mucosa and cecal fermentation in rats. Journal of Nutrition Science and Vitaminology 44, 103-112.

Langhendries JP, Detry J, Van Hees J, Lamboray JM, Darimont J, Mozin MJ, Secretin MC \& Senterre J (1995) Effect of a fermented infant formula containing viable bifidobacteria on the fecal flora composition and $\mathrm{pH}$ of healthy full-term infants. Journal of Pediatric Gastroenterology and Nutrition 21, $125-129$.

Hamosh M (2001) Bioactive factors in human milk. Pediatric Clinics in North America 48, 69-86.

Midtvedt T (1999) Microbial functional activities. In Probiotics, Other Nutritional Factors and Intestinal Microflora, volume 42, pp. 79-97 [LA Hanson and RH Yolken, editors]. Nestlé Nutrition Workshop Series Philadelphia: Lippincott-Raven Publishers.

Moro G, Minoli I, Mosca F, Jalinek J, Stahl B \& Boehm G (2001) Dosage effect of oligosaccharides on faecal flora and stool characteristics in term infants. Journal of Pediatric Gastroenterology and Nutrition 32, 401.

Nakhla T, Fu D, Brodsky NL \& Hurt H (1999) Natural oligosaccharide content of preterm human milk. British Journal of Nutrition 82, 333-335.

Newburg DS (1996) Oligosaccharides and glycoconjugates in human milk: their role in host defence. Journal of Mammary Gland Biology and Neoplasia 1, 271-283.

Picciano MF (2001) Nutrient composition of human milk. Pediatric Clinics in North America 48, 53-67.

Rigo J, Pielman C, Studzinski F, Knol J \& Bindels JG (2001) Clinical evaluation in term infants of a new formula based on prebiotics $\beta$-palmitate and hydrolysed proteins. Journal of Pediatric Gastroenterology and Nutrition 32, 402.

Saavedra J (2000) Probiotics and infectious diarrhea. American Journal of Gastroenterology 95, suppl 1, S16-S18.

Saavedra JM, Tschernia A, Moore N, Abi-Hanna A, Coletta F, Emenhiser C \& Yolken RH (1999) Gastro-intestinal function in infants consuming a weaning food supplemented with oligo-fructose, a prebiotic. Journal of Pediatric Gastroenterology and Nutrition 29, A95.

Sabharwal H, Sjoblad S \& Lundblad A (1991) Sialyted oligosaccharides in human milk and feces of pretrem, full-term, and weaning infants. Journal of Pediatric Gastroenterology and Nutrition 12, 480-484.

Taranto MP, Medici M, Perdigon G, Ruiz Holgado AP \& Valdez GF (2000) Effect of Lactobacillus reuteri on the prevention of hypercholesterolemia in mice. Journal of Dairy Science 83, 401-403.

Tschernia A, Moore N, Abi-Hanna A, Yolken RH, Coletta F, Emenhiser C \& Saavedra JM (1999) Effects of long-term consumption of a weaning food supplemented with oligofructose, a prebiotic, on general infant health status. Journal of Pediatric Gastroenterology and Nutrition 29, A58.

Young J (1998) European market developments in prebiotic- and probiotic-containing foodstuffs. British Journal of Nutrition $\mathbf{8 0}$, suppl, 231-233. 